\title{
Acknowledgement to Referees
}

Received: 18 December 2012/ Accepted: 20 December 2012/Published online: 6 January 2013

(C) Springer-Verlag Berlin Heidelberg 2013

The Editors highly appreciate the essential support provided by the following colleagues, who have been kind enough to review manuscripts for this journal in the last year:

Ainsbury Elizabeth
Alberti Angelo
Allemand Isabelle
Ambrozova Iva
Azizova Tamara
Baffa Oswaldo
Barjaktarovic Zarko
Belli Mauro
Belyaev Igor
Beresford Nicholas
Bijwaard Harmen
Blanchardon Eric
Blomgren Klas
Bolch Wesley
Borgmann Kerstin
Bortolussi Silva
Bottollier-Depois JF
Brown Justin
Campos Marcia Pires
Chang Fu-Kei
Chankova Stephka G
Ciesielski Bartlomiej
Cullings Harry Michael
da Costa Lauria Dejanira
Dauer Lawrence T
Dekkers Fieke
de Micco Veronica
Dikomey Ekkehard
Dollinger Günther
dos Santos Alan Roger
Druid Henrik
Dumont Francis
Dziegielewski Jaroslaw
Eckermann Keith
Emfietzoglou Dimitris
Faião-Flores Fernanda
Fuma Shoichi
Furukawa Kyoji
Geras'kin Stanislav A

Ainsbury Elizabeth

Alberti Angelo

Ambrozova Iva

Azizova Tamara

Baffa Oswaldo

Barjaktarovic Zarko

Belli Mauro

Beresford Nicholas

Bijwaard Harmen

Eric

Borgmann Kerstin

(

作

Campos Marcia Pires

Chang Fu-Kei

Chankova Stephka G

Ciesielski Bartlomiej

Cullings Harry Michael

da Costa Lauria Dejanira

Dekkers Fieke

de Micco Veronica

Dikomey Ekkehard

Dollinger Günther

dos Santos Alan Roger

Druid Henrik

Dumont Francis

Dziegielewski Jaroslaw

Eckermann Keith

Emfietzoglou Dimitris

Faião-Flores Fernanda

Furukawa Kyoji

Geras'kin Stanislav A
Gering Florian

Gudowska-Nowak Ewa

Harley Naomi

Hofmann Werner

Hopewell John

Horneck Gerda

Hunter Nezahat

Idle Jeff

Imanaka Tetsuji

Ivashkevich Alesia

Jones Bleddyn

Kadhim Munira

Karabulut Bünyamin

Kennedy Ann

Kiefer Jürgen

Kinashi Yuko

Kucuk Nil

Kurudirek Murat

Laurier Dominique

Lee Chun Ma

Lee Yong Woo

Leitgeb Norbert

Limoli Charles L

Linard Christine

Linares Vidal Victoria

Little Mark P

Lund Anders

Maghraby Ahmad

Mahmoud Sherif Siddick

Manohara SR

Manti Lorenzo

McFarland Hugh

McMahon Stephen

Milligan Jamie

Müller Wolfgang U

Oeh Uwe

Oskolkov Borys

Pawar Pravina Prakash

Pawel David
Petoussi-Henss Nina

Phan Nghi

Pinto Marcela MPL

Popov-Celeketic Dusan

Prise Kevin

Real Almudena

Rithidech Kanokporn Noy

Rosemann Michael

Rothkamm Kai

Ruebe Claudia

Ruiz Gomez Miguel

Saito Kimiaki

Saito Masatoshi

Sakashita Tetsuya

Sakata Ritsu

Sargentini Neil

Sazykina Tatiana

Scarpato Roberto

Schmid Ernst

Schmid Thomas E

Schöllnberger Helmut

Schwarcke Marcelo

Sevilla Michael

Sigurdson Alice

Shetty Pramod

Shore Roy

Shuryak Igor

Slade Dea

Smith Graham

Sokolov Mykyta

Soriano Jose

Spix Claudia

Stewart Fiona

Synal Hans-Arno

Tabet Eugenio

Takamura Noboru

Tucker James D

Tuner Hassan

Umesh TK 
Unger Kristian

Vandenhove Hildegarde

Veronese Ivan

Voisin Philippe

Wakeford Richard
Walker Mary $\mathrm{P}$

Wilczynski Slawomir

Willmore Elaine

Winters Thomas

Wittig Andrea
Wojcik Andrzej

Yukihara Eduardo

Zakeri Farideh

Zoelzer Friedo 